\title{
Lower Bounds on Moving a Ladder in Two and Three Dimensions*
}

\author{
Yan Ke and Joseph O'Rourke \\ Department of Computer Science, Johns Hopkins University, \\ Baltimore, MD 21218, USA
}

\begin{abstract}
It is shown that $\Omega\left(n^{2}\right)$ distinct moves may be necessary to move a line segment (a "ladder") in the plane from an initial to a final position in the presence of polygonal obstacles of a total of $n$ vertices, and that $\Omega\left(n^{4}\right)$ moves may be necessary for the same problem in three dimensions. These two results establish lower bounds on algorithms that solve the motion-planning problems by listing the moves of the ladder. The best upper bounds known are $O\left(n^{2} \log n\right)$ in two dimensions, and $O\left(n^{5} \log n\right)$ in three dimensions.
\end{abstract}

\section{Introduction}

The problem of moving a ladder in two dimensions has attracted considerable attention. The problem is to find a sequence of motions, if they exist, that will move the ladder from a given initial position to a given final position without penetrating known polygonal obstacles with a total of $n$ vertices. A ladder is an oriented line segment; it is a one-dimensional object. After the pioneering work of Schwartz and Sharir [9] established that the problem can be solved in polynomial $\left(O\left(n^{5}\right)\right)$ time, several nearly quadratic algorithms were developed [6], [7], [5], [12], at least one of which [5] can probably be reduced to exactly quadratic, $O\left(n^{2}\right)$ for polygonal obstacles with a total of $n$ vertices. Sharir posed the problem of establishing a lower bound on the number of moves that might be necessary in 1985 [11], as no example was known to require more than a linear number of moves. This paper constructs a set of obstacles that force $\Omega\left(n^{2}\right)$

* This work was partially supported by NSF Grants DCR-83-51468 and grants from Martin Marietta, IBM, and General Motors. 
moves between a certain initial and final position, thereby settling the worst-case time complexity of any algorithm that lists the moves. The construction first appeared in [8].

The same problem with three-dimensional obstacles (but still a onedimensional ladder) was studied in the fifth Schwartz and Sharir piano movers' paper, which established an $O\left(n^{11}\right)$ upper bound [10]. Recently we proposed an $O\left(n^{6} \log n\right)$ algorithm [3], [4], and independently Canny obtained an $O\left(n^{5} \log n\right)$ algorithm as a specialization of a more general result [1]. The two-dimensional lower-bound example in [8] does not extend directly to three dimensions. We construct in this paper a more complex two-dimensional example that does extend naturally (although not trivially), and establish that $\Omega\left(n^{4}\right)$ moves are necessary for this extension.

A gap remains between the lower bound of $\Omega\left(n^{4}\right)$ and the best upper bound of $O\left(n^{5} \log n\right)$. We suspect the lower bound is closer to the truth.

We first discuss the definition of "simple move," then establish the twodimensional lower bound via two examples, one easy to understand but not extensible to three dimensions, and another that is more delicate but does extend. Finally we describe the three-dimensional construction and argue for its correctness.

\section{Simple Moves}

For the two-dimensional problem, the position of the ladder can be considered a point in a three-dimensional "configuration space" of free positions: two dimensions to represent the translation of one endpoint of the ladder, and one dimension to represent its orientation. For the three-dimensional problem, the position of the ladder can be represented by a point in a five-dimensional space: three dimensions of translation, and two for orientation. Let $S$ be the relevant configuration space for the problem. A continuous motion of the ladder is then a path in $S$. We say a motion is simple if it may be represented by an algebraic curve in $S$ of constant degree $r$. The reason that we choose this definition of what constitutes a simple move is that the algorithms proposed for solving the ladder motion-planning problems naturally output these types of moves. The actual value of $\boldsymbol{r}$ is not important as long as it is a constant independent of $\boldsymbol{n}$. For the purposes of intuition, it is convenient to think of $r=1$, when a simple move cannot reverse direction in any dimension. Higher values of $r$ permit reversal, but the number of reversals is limited by $r$, because the number of roots of an algebraic equation is limited by the degree of the equations.

Note that the definition of "simple" is dependent on the coordinate system chosen for $S$. This seems unavoidable. Our bounds hold for any coordinate system that represents the translational components of the ladder's motion in the natural Cartesian coordinate system.

The examples described in the following sections will establish lower bounds on the number of simple moves. 


\section{First Two-Dimensional Construction}

Leven and Sharir observed [5] that the number of connected components of configuration space may be $\Omega\left(n^{2}\right)$. Their construction is shown in Fig. 1: two rows of poles $A$ and $B$ between barriers Top and Bot are easily seen to create $\Omega\left(n^{2}\right)$ mutually inaccessible positions for a long ladder. This example establishes a lower bound for any algorithm that constructs all connected components of the configuration space; but only the component containing the initial position needs to be constructed. All algorithms following the cell decomposition or retraction paradigms of which we are aware do construct all connected components, but it is conceivable that sweeping or "wavefront expansion" techniques might only construct the one necessary component. So Fig. 1 is insufficient to establish a lower bound on all conceivable algorithms.

We now describe the example from [8], but only at a high level. Understanding the basic mechanism is important for the remainder of the paper, but as we will supplant this example with another later, there is no need to specify the details of the construction.

The example is a direct generalization of Fig. 1, except that (a) the $\Omega\left(n^{2}\right)$ mutually inaccessible positions of the ladder are made accessible to one another, and (b) in such a way that the accessibility graph between positions is a chain: the last position is accessible from the first only by passing through each intermediate position. An instance of the design is shown in Fig. 2. It consists of six components: three rows of poles, $A, B$, and $C, n+1$ poles per row, bottom and top barriers Bot and Top, and "spikes" emanating from Top. The $A$ and $B$ poles correspond in function to the $A$ and $B$ poles in Fig. 1. Let $L$ be the ladder and $|L|$ its length. Both the vertical distance between the $C$ poles and Top, and between $B o t$ and the $A$ poles is $|L|+\varepsilon$. Thus when the ladder is abutting on Top it is free of the $C$ poles, and when abutting on $B o t$ it is free of the $A$ poles. But aside from brief excursions into spikes, it is always confined between two $B$ poles.

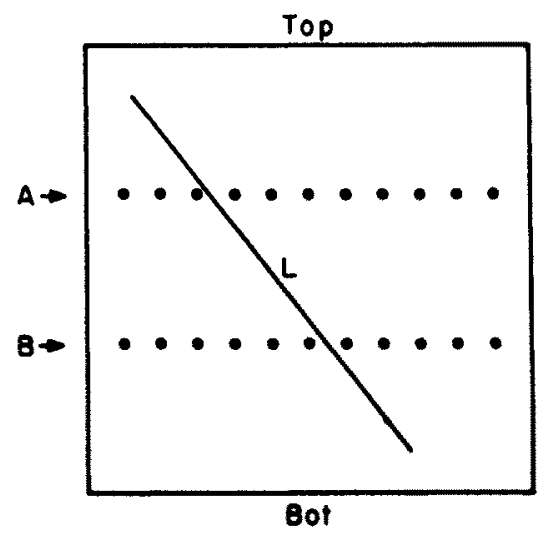

Fig. 1. A set of obstacles that define $n^{2}$ mutually inaccessible positions. 


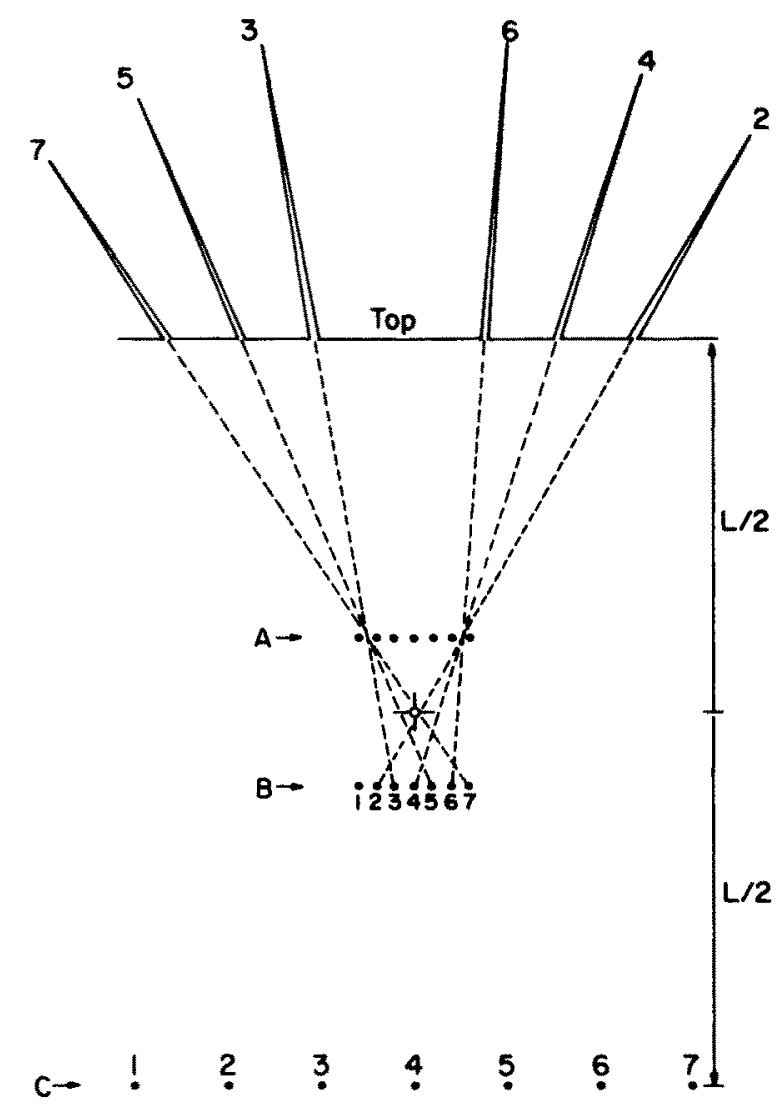

Bot

Fig. 2. A design that requires $n^{2}$ moves to reposition a ladder of length $L$.

Let $A_{i}$ and $B_{i}$ represent open segments between the $i$ th and $(i+1)$ th poles on the $A$ and $B$ row, respectively. Say that $L$ is in position $\left(A_{i}, B_{j}\right)$ if it intersects both $A_{i}$ and $B_{j}$. The ladder's initial position is $\left(A_{1}, B_{1}\right)$ and the final position is $\left(A_{n}, B_{n}\right)$. Thus the ladder must hop over each $B$ pole from 1 to $n$. Because the Top and $B o t$ barriers constrain the ladder within the $B$ row, the only way a $B$ pole can be jumped is to move the ladder into a spike. Each spike is extremely thin, and only permits entrance to a ladder with a specific $\left(A_{i}, B_{j}\right)$ position. In order to jump from $B_{1}$ to $B_{2}$, the ladder must have position $\left(A_{n}, B_{1}\right)$; then it can slide into the spike, turn slightly, and slide out in position $\left(A_{n}, B_{2}\right)$. In order to jump from $B_{2}$ to $B_{3}$, the ladder must have position $\left(A_{1}, B_{2}\right)$; it can then move to $\left(A_{1}, B_{3}\right)$. To jump from $B_{3}$ to $B_{4}$, it must be positioned at $\left(A_{n}, B_{3}\right)$. And so 
on. Note that the jumps of the even $B$ poles require the ladder to be in $A_{1}$, and the jumps of the odd $B$ poles require it to be in $A_{n}$. Thus for each $B$ transition, the ladder must move through the entire $A$ row. This is precisely where $\Omega\left(n^{2}\right)$ moves are forced.

With the ladder fixed in $B_{j}$, the ladder can be advanced through the $A$ row by moving it down until touching Bot, then rotating as far as is permitted by the $B$ and $C$ poles. The spacing of the poles is designed so that this move jumps no more than a constant number of $A$ poles; for the design in Fig. 2, this constant is 4. (This claim is supported in [8].) To advance further in the $A$ row, the constraining $C$ pole must be jumped. This is accomplished by moving the ladder up until touching Top, then rotating as far as is permitted by the $A$ and $B$ poles. The $C$ poles are spaced such that just one pole can be jumped by this maneuver.

Assume that $r=1$, so that each reversal in direction initiates a distinct simple move. Then each advance of four poles in the $A$ row requires two reversals in the translational component of the ladder's position, and so two simple moves, and each advance of one pole in the $B$ row requires the entire $A$ row to be traversed, plus a move into the spike. So the total number of simple moves required from the initial position $\left(A_{1}, B_{1}\right)$ to the final position $\left(A_{n}, B_{n}\right)$ is at least $n(2\lceil n / 4\rceil+1)=\Omega\left(n^{2}\right)$. Since there are only $O(n)$ vertices in the entire design, this establishes the claimed lower bound for $r=1$. It is clear that larger values of $r$ simply divide the number of moves by a constant, so the lower bound holds independent of $r$.

\section{An Extensible Two-Dimensional Design}

The three-dimensional lower-bound construction is essentially an orthogonal Cartesian product of the pattern in Fig. 2. The $A$ and $B$ rows of $\Theta(n)$ holes become $A$ and $B$ planes of $\Theta\left(n^{2}\right)$ holes (formed with $\Theta(n)$ beams crossing in a latticework arrangement). A straightforward mixing of two copies of Fig. 2, however, leads to $\Theta\left(n^{2}\right)$ spikes, so that even though $\Omega\left(n^{4}\right)$ moves are required, the input size is $N=\Theta\left(n^{2}\right)$, so that only a bound of $\Omega\left(N^{2}\right)$ is established. This necessitates designing a more complex two-dimensional lower-bound example, which, however, generalizes more easily to three dimensions.

The main idea is the same as in Fig. 2, but without the troublesome spikes. The effect of the spikes is now achieved by replacing Top in Fig. 2 with a $Z$ row of holes where Top was, and a new Top barrier where the tips of the spikes reached. In effect, the sides of the spikes are removed. See Fig. 3. Now a crucial property of the spikes is lost: the sides of the spikes ensured that $L$ could only slide in if it were oriented precisely. In order to guarantee that this property still holds without the restraining sides, several other modifications are made.

First, rather than rows of poles, we now use rows of segments with hole gaps. This gives us more control over the size of the holes. Second, the holes are not all the same size (in the $B$ and $Z$ rows in particular). Third, and most importantly, the holes are arranged to satisfy a collection of visibility constraints. Let $A_{i}, B_{j}$, 


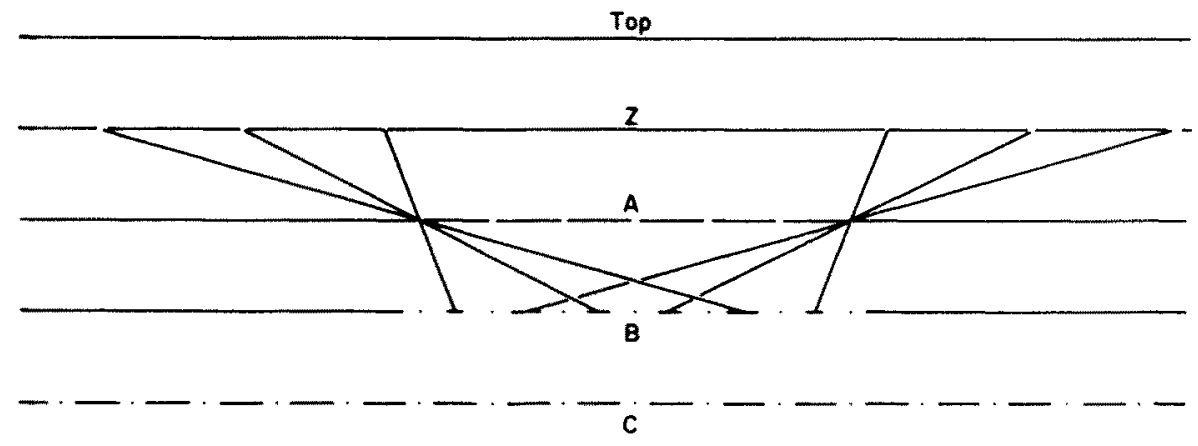

Bot

Fig. 3. A two-dimensional example that requires $n^{2}$ moves, and is extensible to three dimensions. The horizontal scale in this figure is greatly magnified. The rightmost and leftmost reference points on the $C$ row are not shown.

$C_{k}$, and $Z_{l}$ be holes on the $A, B, C$, and $Z$ rows. The simplest constraint defines what can be seen from $C_{k}$, through the $B$ holes, onto the $A$ row: for a given $C_{k}$ and $B_{j}$, only $A_{i-1}, A_{i}$, and $A_{i+1}$ must be visible. This ensures that $L$ can skip only at most one $A$ hole at a time, when moving from $A_{i-1}$ to $A_{i+1}$.

To specify this constraint more precisely, we introduce some notation, and make some assumptions. Let $a_{i}, b_{j}, c_{k}$, and $z_{l}$ be reference points inside holes $A_{i}$, $B_{j}, C_{k}$, and $Z_{l}$. The reference points in each row are evenly spaced, with separation 1 in the $A, B$, and $C$ rows, and separation 2 in (each half of) the $Z$ row:

$$
\left|a_{i+1}-a_{i}\right|=\left|b_{j+1}-b_{j}\right|=\left|c_{k+1}-c_{k}\right|=1
$$

and $\left|z_{1+1}-z_{l}\right|=2$. The extent of each hole is specified by a right and left offset with respect to the reference point. The right and left offsets for $A_{i}$ are $\left(\alpha_{i}, \alpha_{i}^{\prime}\right)$. Thus $A_{i}=\left\{x \mid a_{i}-\alpha_{i}^{\prime} \leq x \leq a_{i}+\alpha_{i}\right\}$. Similarly, the offsets for $B_{j}, C_{k}$, and $Z_{l}$ are $\left(\beta_{j}, \beta_{j}^{\prime}\right),\left(\gamma_{k}, \gamma_{k}^{\prime}\right)$, and $\left(\zeta_{i}, \zeta_{i}^{\prime}\right)$.

For all $a_{i}$ and $b_{j}$ we require a $c_{k}$ collinear with $a_{i}$ and $b_{j}$. If there are $n A$ and $B$ holes, this is easily achieved by having $3 n-2 C$ holes. When $L$ is in $B_{j}$ and $C_{k}$, it must be able to swing from $A_{i}$ to $A_{i \pm 1}$, but it should not be able to reach $\boldsymbol{A}_{i \pm 2}$. Under the assumption that the rows of holes are separated by equal distance (in fact $|L| / 3$ ), the constraint that $A_{i \pm 1}$ is reachable is equivalent to

$$
\begin{aligned}
& \gamma_{k}^{\prime}+2 \beta_{j}+\alpha_{i+1}^{\prime} \geq 1, \\
& \gamma_{k}+2 \beta_{j}^{\prime}+\alpha_{i-1} \geq 1,
\end{aligned}
$$

and that $A_{i \pm 2}$ is unreachable is

$$
\begin{aligned}
& \gamma_{k}^{\prime}+2 \beta_{j}+\alpha_{i+2}^{\prime}<2, \\
& \gamma_{k}+2 \beta_{j}^{\prime}+\alpha_{i-2}<2 .
\end{aligned}
$$




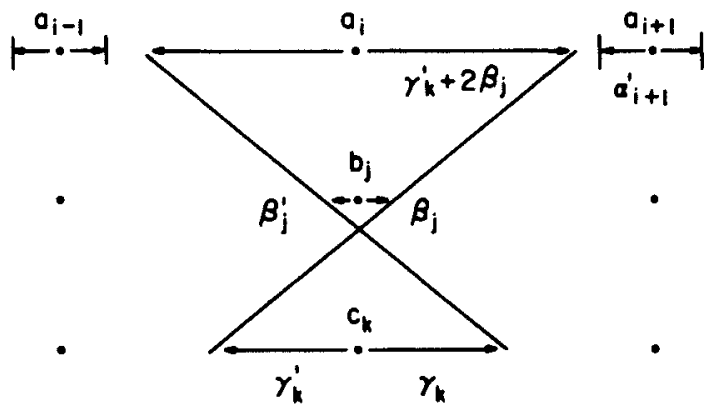

Fig. 4. Constraints between the $A, B$, and $C$ holes. The dots represent reference points on the $A$, $B$, and $C$ rows.

See Fig. 4 for a derivation of the first pair of these equations. Although not $a$ priori clear, it will turn out that our constraints may be satisfied with uniformly sized holes on the $A$ and $C$ rows. Thus we set $\alpha_{i}^{\prime}=\alpha_{i}=\alpha$ for all $i$ and $\gamma_{k}^{\prime}=\gamma_{k}=\gamma$ for all $k$. The above equations are clearly satisfied if

$$
\begin{aligned}
& \gamma+2 \beta_{\text {min }}+\alpha \geq 1, \\
& \gamma+2 \beta_{\text {max }}+\alpha<2,
\end{aligned}
$$

where $\beta_{\min }$ and $\beta_{\max }$ are the minimum and maximum values of $\beta_{j}$ over all $j$.

The more complex constraints specify what can be seen from $Z_{l}$, through the $A$ holes, onto the $B$ row. We first explain these constraints informally. From $Z_{l}$, through $A_{1}$ only, $B_{j}$ and $B_{j+1}$ are simultaneously visible. This means that if $L$ is at $\left(A_{1}, B_{j}\right)$, it can move one $s$ ' $p$ in the $B$ row. To move the next step from $B_{j+1}$ to $B_{j+2}, L$ will have to orient through $A_{n}$. This is achieved by separating the $Z$ holes into left and right halves. This mimics the even/odd alternation achieved by the spikes. But now we must arrange that from $Z_{l}$, through $A_{i}$ where $1<i<n$, no two adjacent $B$ holes can be seen. Otherwise $L$ could advance in the $B$ row without being oriented precisely through $A_{1}$ or $A_{n}$. This is accomplished by distorting the size of the $Z$ and $B$ holes, so that both the odd $B$ holes and the left $Z$ holes diminish in size from left to right, and both the even $B$ holes and the right $Z$ holes increase in size from left to right. Now suppose $Z_{l}$ can just see both $B_{j}$ and $B_{j+1}$ through $A_{1}$, for some odd $j$. Because the odd $B$ holes diminish in size, the separation between an odd hole and its neighbor increases, so that $Z_{l}$ cannot see two adjacent $B$ holes through $A_{2}, A_{3}, \ldots$ Thus $Z_{l}$ is only effective for hopping from $B_{j}$ to $B_{j+1}$ when $L$ is oriented through $A_{1}$.

We now formalize the preceding discussion. Let $0<s_{1}<s_{2}<\cdots<s_{n / 2}<1$ be a series of real numbers that represent the lengths of the solid (opaque) segments of the $B$ row, interleaved as shown in Fig. 5: the segments separating $B_{j}$ and $B_{j+1}$ for odd $j$ get larger, and those for even $j$ smaller, from left to right. Again, although not clear a priori, we can choose

$$
\begin{aligned}
& \beta_{2 j-1}=\beta_{2 j}^{\prime}=\left(1-s_{j}\right) / 2 \quad \text { for } j=1, \ldots, n / 2, \\
& \beta_{2 j}=\beta_{2 j+1}^{\prime}=\left(1-s_{n / 2+1-j}\right) / 2 \text { for } j=1, \ldots, n / 2-1 .
\end{aligned}
$$




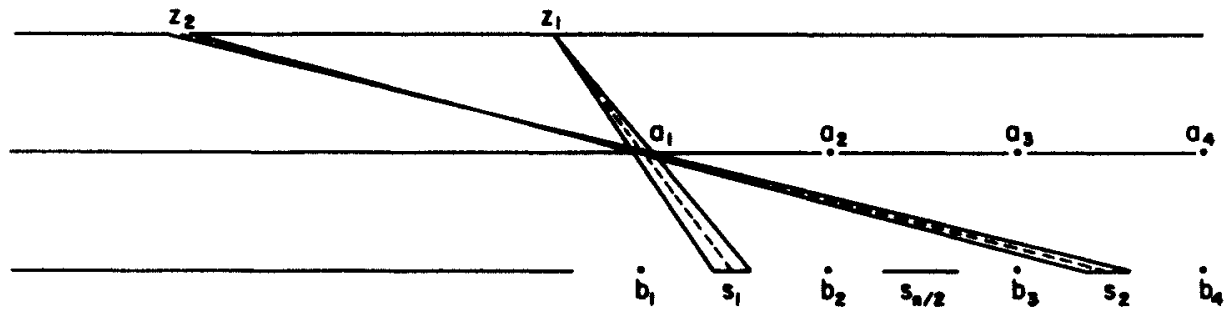

Fig. 5. Reference points and segments in the $B$ row.

The left set of $z_{j}$ reference points, which are numbered from right to left, are chosen to be collinear with $a_{1}$ and $\left(b_{2 j-1}+b_{2 j}\right) / 2$, which is the midpoint of $s_{j}$, for $j=1, \ldots, n / 2$, and similarly the right $Z$ reference points are chosen collinear with $a_{n}$ and $\left(b_{2 j}+b_{2 j+1}\right) / 2$. To permit $L$ to hop from $B_{2 j-1}$ to $B_{2 j}$ when in $A_{1}$, we set

$$
\zeta_{j}=\zeta_{j}^{\prime}=s_{j} / 2-2 \alpha \text {. }
$$

This lets $L$ move a distance of exactly $s_{j}$ in row $B$, which is the separation between the holes, and no more; see Fig. 5. Note that if $L$ is in $B_{2 j-1}$ and $A_{i}, i>1$, it would reach the $Z$ row at a location to the right of $Z_{j}$. Equation (4) and the fact that the $s_{j}$ 's form an increasing sequence imply that the distance $L$ could hop would then be insufficient to reach $B_{2 j}$. Another way to look at this crucial property, which we will use in the three-dimensional construction, is as follows. Imagine each $Z$ hole to be a line segment light source. Figure 5 shows that the light the $Z$ holes cast through the $A_{1}$ hole onto the $B$ row, precisely coincides with the opaque segments $s_{1}, s_{2}, s_{3}, \ldots$. The light the $Z$ holes cast through any other hole $A_{i}, i>1$, forms the same pattern, but shifted $2(i-1)$ units rightward. Thus the light from $Z_{1}$ projects to a length of $s_{1}$, but this falls on an opaque segment of length $s_{i}$, and since $s_{1}<s_{i}$, it is too small to connect the adjacent holes on the $B$ row separated by $s_{i}$. Thus the shifted pattern nests inside the unshifted pattern because of the monotonic increase in the $s_{i}$ sequence, ensuring its ineffectiveness.

There is one further constraint. In order to keep the $Z$ holes separated, we must have $\zeta_{j}+\zeta_{j+1}^{\prime}<2$ and, on the other hand, the $Z$ holes must have positive length. Equation (4) permits these constraints to be written as

$$
0<s_{\min } / 2-2 \alpha<s_{\max } / 2-2 \alpha<1 \text {. }
$$

Equations (1)-(5) represent a series of constraints on the dimensions of the holes: equations (1) and (2) interrelate the $\alpha, \beta$, and $\gamma$ dimensions, and equations (5), (3), and (4) relate $\alpha$ to $s_{i}, \beta$ to $s_{i}$, and $\gamma$ to $s_{i}$, respectively. Although it is not clear that there must be a solution to these constraint equations, Fig. 3 illustrates a solution. If we let the $s_{i}$ sequence be $0.2,0.3,0.4$, then $s_{\min }=0.2$ and $s_{\max }=0.4$, and equation (3) implies that $\beta_{\max }=0.4$ and $\beta_{\min }=0.3$. If we further choose $\alpha=0.04$ and $\gamma=0.4$, then the constraints of equations (1), (2), and (5) are satisfied. Figure 3 was designed with these parameters, which will be used again in the three-dimensional design. 
Finally, it should be mentioned that the horizontal scale in Figs. 3 and 5 is greatly magnified: it is necessary to squash the design horizontally so that the rotation of the ladder is negligible, negligible in the sense that the rotation is insufficient to permit the ladder to fit properly between two rows of Fig. 3 that are separated by $2|L| / 3$. A bound of $\cos ^{-1}(2 / 3)=48.2^{\circ}$ on the deviation from the vertical suffices, and is easily achieved.

\section{Three Dimensions}

\subsection{Coarse Description}

We now come to the three-dimensional construction. We first describe it informally before plunging into the details, which are a bit complicated. The main idea is to replicate Fig. $3 n$ times, roughly mixing two copies orthogonally. The absence of spikes allows the mixing to be accomplished with $\Theta(n)$ polyhedron vertices, avoiding the problem that arises with Fig. 2 . The construction consists of six parallel planes, each an exact counterpart of a row in Fig. 3; see Fig. 6. Planes $A$ and $B$ each have $\Theta\left(n^{2}\right)$ holes, determining $\Theta\left(n^{4}\right)$ positions of the ladder. As before, we design the environment so that these positions are accessible to one another only through a single path, depicted in Fig. 7(a) and (b): the ladder must traverse every hole on the $A$ plane in the snaking path shown in Fig. 7 (a) for each step in the $B$ plane; the path in the $B$ plane is a similar path. ${ }^{1}$ The design of the holes on the $A$ plane is shown in Fig. 8 . As before, movement

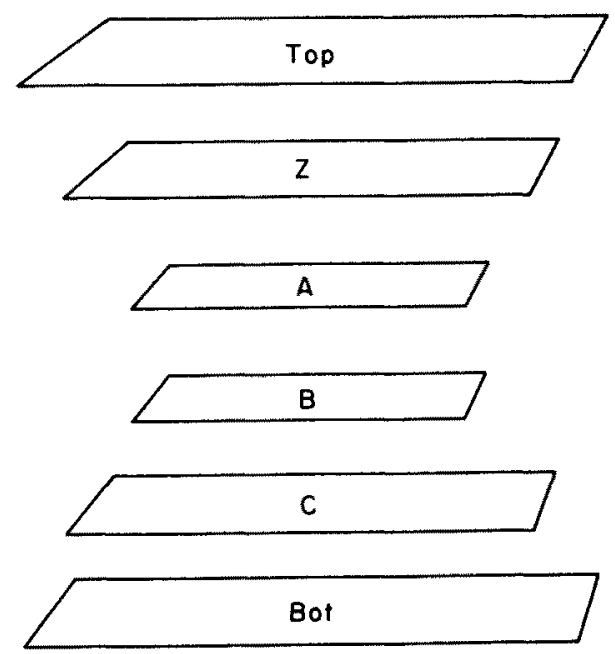

Fig. 6. The planes of the three-dimensional design.

\footnotetext{
'In [3] we chose to make these two paths orthogonal, but the design is a little simpler if they are parallel.
} 


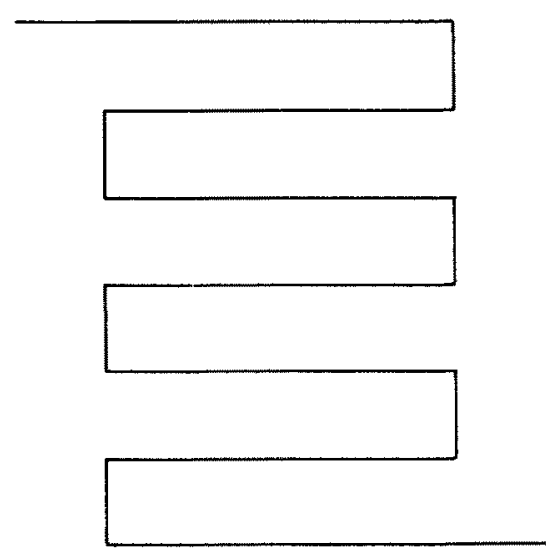

(a)

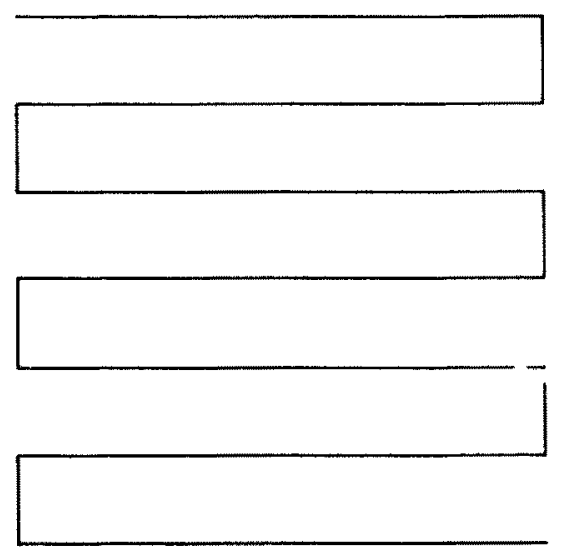

(b)

Fig. 7. Paths on the $A(a)$ and $B(b)$ planes. $A_{11}$ and $B_{11}$ are the upper left endpoints of each path.

through the $A$ plane is effected by sliding the ladder down to touch Bot through the auxiliary $C$ plane, shown in Fig. 9. A set of visibility constraints ensure that each $C$ hole can only see at most three horizontally adjacent $A$ holes through the $B$ plane. This forces motion through the $A$ plane to be horizontal. But notice that the extreme holes to the right and left on the $A$ plane are stretched vertically; this permits the horizontal pieces of the path to link up as in Fig. 7(a). The result is that, with the ladder through any fixed $B$ hole, it can move from $A_{11}$ to $A_{n n}$ in $\Theta\left(n^{2}\right)$ moves, where $A_{i j}$ is the hole in the $i$ th row and $j$ th column on the $A$ plane.

As in two dimensions, the design of the $B$ and $Z$ plane holes is more complicated. As before, the $Z$ holes are partitioned into two groups, one to be used when $L$ is in $A_{11}$, and the other when in $A_{n n}$. And as before, the two groups 


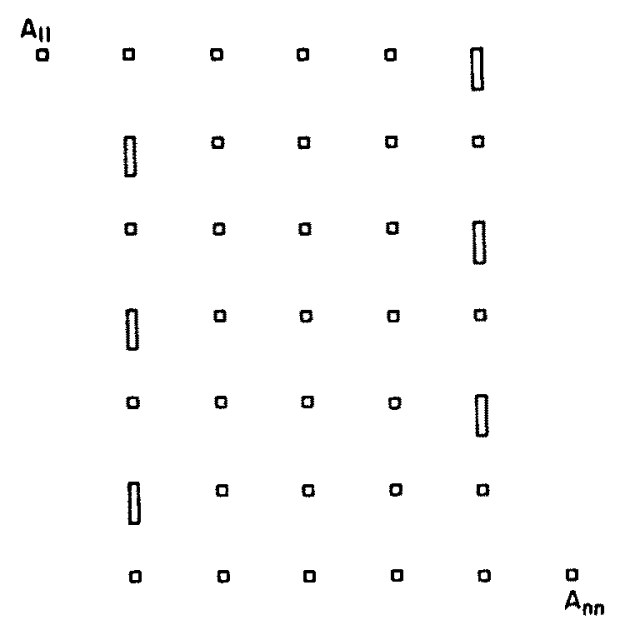

Fig. 8. The $A$ plane.

effectively link only every other $B$ hole, forcing complete traversal of the $A$ plane between every two moves on the $B$ plane. The mechanism is essentially the same: the size of both the $Z$ and $B$ holes vary in a systematic manner so that a particular left $Z$ hole is only effective for connecting two horizontally adjacent $B$ holes when the ladder is through $A_{11}$. The same $Z$ hole cannot see two adjacent $B$ holes through any other $A$ hole because of the size variation. An exception occurs with the first column of $A$ holes, which is handled by offsetting $A_{11}$ as shown in Fig. 8, leaving $A_{11}$ the only hole in the first column. All these constraints can be expressed with a collection of visibility equations, similar to those detailed in the previous section, whose solution leads to the $B$ plane design shown in Fig. 10, and the $Z$ plane shown in Fig. 11.

\subsection{The Design in Three Dimensions in Detail}

It seems unfortunately necessary to describe the construction in some detail in order to establish that it works as described. Avoiding "shortcuts" in the paths in Fig. 7 is a delicate affair, and requires an argument. The reader uninterested in the details can skim the equations below and depend on the figures for intuition.

5.2.1. Notation. The four planes $A, B, C$, and $Z$, are parallel and separated by $|L| / 3$. Let $A_{i j}, B_{i j}, C_{i j}$, and $Z_{i j}$ represent the holes (as sets) on the planes in the $i$ th row and $j$ th column. (We will not try to use distinct subscripts for each plane.) Each hole is a rectangle. Let $a_{i j}, b_{i j}, c_{i j}$, and $z_{i j}$ be reference points inside the holes $A_{i j}, B_{i j}, C_{i j}$, and $Z_{i j}$, respectively. These reference points are not necessarily in the center of the holes; our design will place them in the center on the $A$ and $C$ planes. The reference points are evenly spaced on all four planes, with 


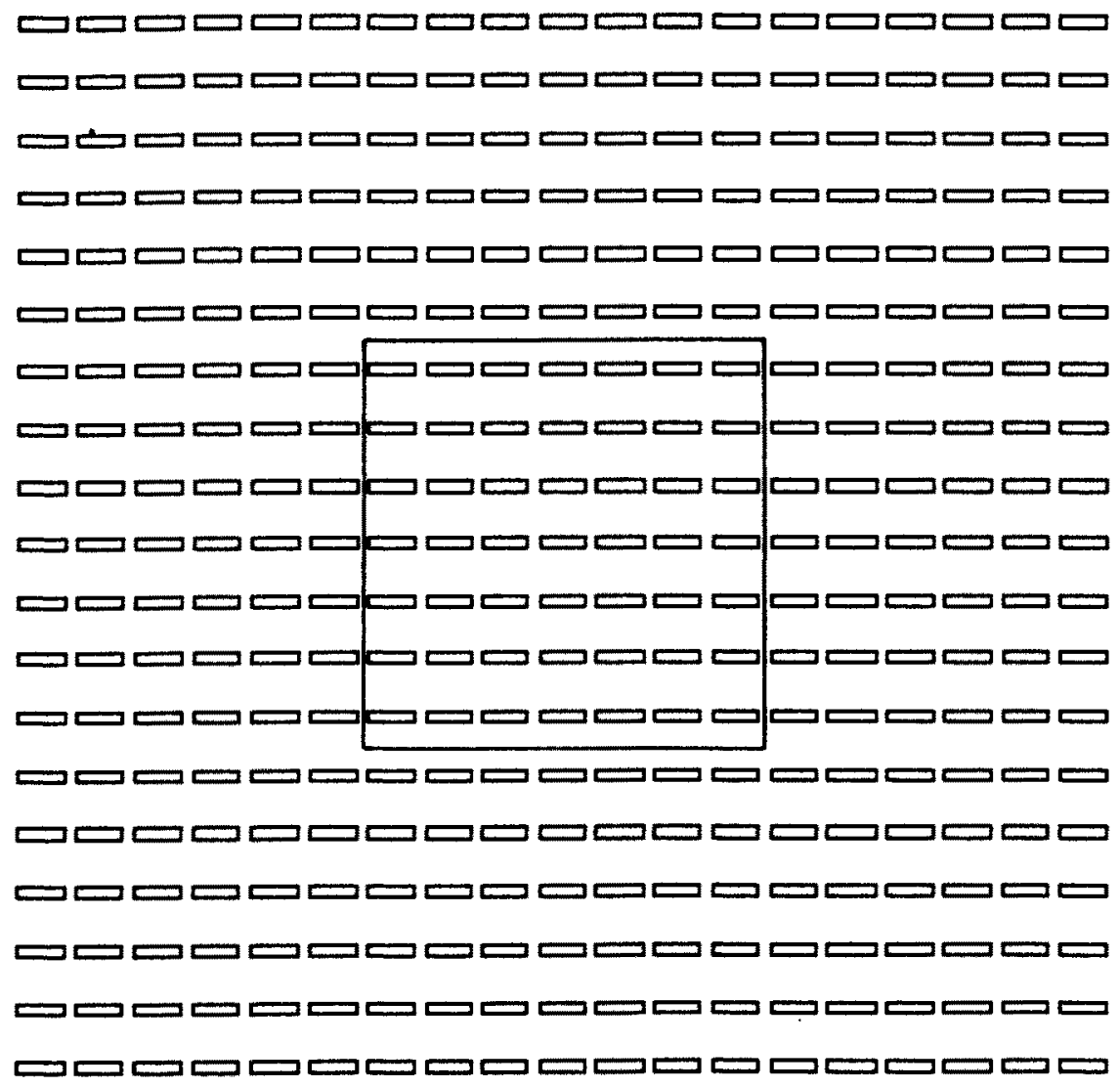

Fig. 9. The $C$ plane. The outline of the square containing the $A$ holes is shown to establish scale.

separation 1 on all but the $Z$ plane, both horizontally and vertically, and a horizontal separation of 2 on the $Z$ plane and 1 vertically:

$$
\begin{gathered}
\left|a_{i+1, j}-a_{i j}\right|=\left|b_{i+1, j}-b_{i j}\right|=\left|c_{i+1, j}-c_{i j}\right|=1, \\
\left|a_{i, j+1}-a_{i j}\right|=\left|b_{i, j+1}-b_{i j}\right|=\left|c_{i, j+1}-c_{i j}\right|=1, \\
\left|z_{i+1, j}-z_{i j}\right|=1 ; \quad\left|z_{i, j+1}-z_{i j}\right|=2 .
\end{gathered}
$$

(There are a few limits on the ranges of indices for which these equations hold, but these will become clear later.)

The extent of each hole is specified by four offsets with respect to its reference point. The four offsets for $A_{i j}$ are $\alpha_{i j}^{(0)}, \alpha_{i j}^{(1)}, \alpha_{i j}^{(2)}, \alpha_{i j}^{(3)}$, where the superscript $(k)$ indicates that the direction of offset is at an angle of $k \pi / 2$ with respect to the horizontal, as shown in Fig. 12. Similarly, the offsets for $B_{i j}, C_{i j}$, and $Z_{i j}$ are $\beta_{i j}^{(k)}$, $\gamma_{i j}^{(k)}, \zeta_{i j}^{(k)}$, respectively, $k=0,1,2,3$. Thus the hole $A_{i j}$ is the set of points

$$
\left\{(x, y) \mid a_{i j}-\alpha_{i j}^{(2)} \leq x \leq a_{i j}+\alpha_{i j}^{(0)}, a_{i j}-\alpha_{i j}^{(3)} \leq y \leq a_{i j}+\alpha_{i j}^{(1)}\right\} .
$$




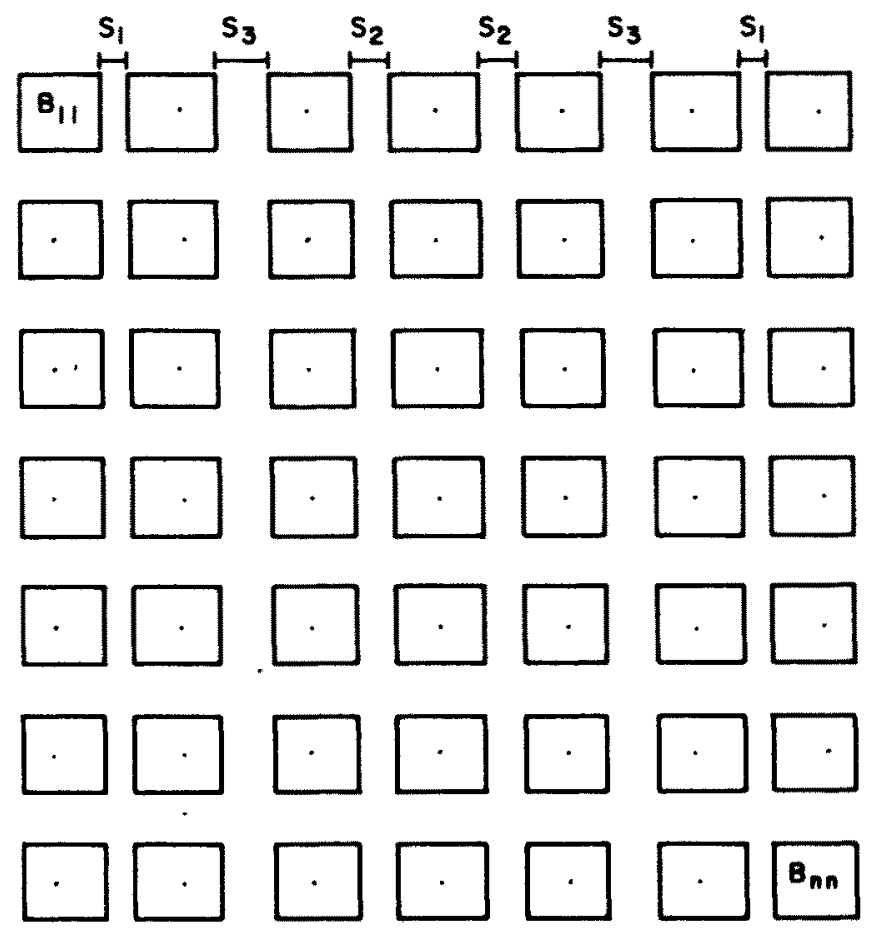

Fig. 10. The $B$ plane. The dots are the $b_{1 y}$ reference points.

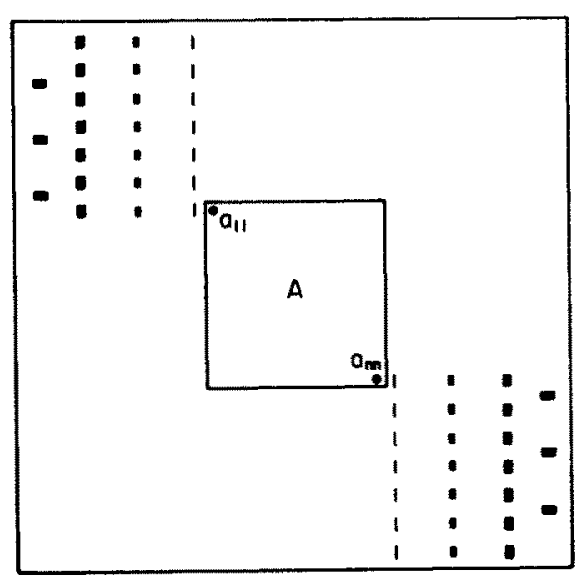

Fig. 11. The $Z$ plane. The outline of the square containing the $A$ holes is shown to establish scale. 


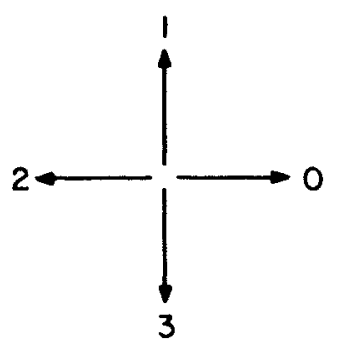

Fig. 12. Definition of direction superscripts.

5.2.2. Design of the $A$ Plane. It will develop that the various constraints may be satisfied with all the holes on the $A$ plane squares, except for $A_{2 i, 2}$ and $A_{2 i-1, n-1}$, $i=1, \ldots, n / 2$. Thus we set

$$
\begin{aligned}
\alpha_{i j}^{(0)} & =\alpha_{i j}^{(2)}=\alpha^{(0)} & & \text { for all }(i, j), \\
\alpha_{i j}^{(1)} & =\alpha_{i j}^{(3)}=\alpha^{(1)}=\alpha^{(0)} & & \text { for all }(i, j) \text { except }(2 i, 2) \text { and }(2 i-1, n-1), \\
\alpha_{2 i-1, n-1}^{(1)} & =\alpha_{2 i, 2}^{(3)}=\alpha^{(1)} & & \text { for } i=1, \ldots, n / 2, \\
\alpha_{2 i-1, n-1}^{(3)} & =\alpha_{2 i, 2}^{(1)}=\alpha^{(3)} & & \text { for } i=1, \ldots, n / 2 .
\end{aligned}
$$

The complete layout of the $A$ plane is shown in Fig. 8 for $n=7$. Note that $A_{11}$ is the only hole in the first column, and $A_{n n}$ is the only hole in the last column. This feature of the design is crucial and will be used in Section 5.3 below.

5.2.3. Design of the $C$ Plane. For each pair of reference points on the $A$ and $B$ planes $a_{i j}$ and $b_{k l}$, we require a hole and reference point $c_{r s}$ on the $C$ plane. Naively this may seem to require $\Theta\left(n^{4}\right)$ holes on the $C$ plane, but in fact $(3 n-2)^{2}$ holes suffice, as illustrated in Fig. 9. We will see that, just as in the two-dimensional construction, the offsets in the $C$ plane may be chosen equal in the horizontal direction, and also in the vertical direction:

$$
\begin{aligned}
& \gamma_{i j}^{(0)}=\gamma_{i j}^{(2)}=\gamma^{(0)}, \\
& \gamma_{i j}^{(1)}=\gamma_{i j}^{(3)}=\gamma^{(1)} .
\end{aligned}
$$

These equations hold for all $(i, j)$. Thus all the holes on the $C$ plane are rectangles centered on the reference points.

The motion of $L$ through the $A$ plane is largely horizontal, as illustrated in Fig. 7(a). When the ladder is moving horizontally through the $A$ plane, the constraints relating the $A, B$, and $C$ hole horizontal dimensions are the same as equations (1) and (2) in the two-dimensional construction:

$$
\begin{aligned}
& \gamma^{(0)}+2 \beta_{\min }^{(0)}+\alpha^{(0)} \geq 1 \\
& \gamma^{(0)}+2 \beta_{\max }^{(0)}+\alpha^{(0)}<2 .
\end{aligned}
$$


Here

$$
\begin{aligned}
& \beta_{\min }^{(0)}=\min _{i, j}\left\{\beta_{i j}^{(0)}, \beta_{i j}^{(2)}\right\}, \\
& \beta_{\max }^{(0)}=\max _{i, j}\left\{\beta_{i j}^{(0)}, \beta_{i j}^{(2)}\right\} .
\end{aligned}
$$

Equation (6) says that even for the smallest hole on the $B$ plane, the $C$ holes are big enough to permit the ladder to move from one $A$ hole to either horizontal neighbor, and equation (7) says that even for the largest hole on the $B$ plane, the $C$ holes do not permit the ladder to move beyond the immediate neighbor.

Vertical motion of the ladder on the $A$ plane is usually forbidden, except when $j=2$ or $j=n-1$, when motion between two vertically adjacent $A$ holes is permitted. These constraints can be phrased as

$$
\begin{aligned}
& \gamma^{(1)}+2 \beta^{(1)}+\alpha^{(1)}<1 \\
& \gamma^{(1)}+2 \beta^{(1)}+\alpha^{(3)} \geq 1 \\
& \gamma^{(1)}+2 \beta^{(1)}+\alpha^{(3)}<2 .
\end{aligned}
$$

Here

$$
\beta^{(1)}=\beta_{\min }^{(1)}=\beta_{\min }^{(3)}=\beta_{\max }^{(1)}=\beta_{\max }^{(3)}
$$

because we will choose to make the $B$ holes uniform in the vertical direction. Equation (8) says that vertical motion in the $A$ plane is not usually possible (when the relevant offset is $\alpha^{(1)}$ ), and equations (9) and (10) are the counterparts of (6) and (7), permitting motion when the larger $\alpha^{(3)}$ is the relevant offset.

5.2.4. The Design of the $B$ and $Z$ Planes. The design of the $B$ plane is the most complex, and must be considered in conjunction with the design of the $Z$ plane. The ladder is to move through the $B$ plane mostly horizontally, as shown in Fig. 7 (b). While it is moving horizontally through a row of $B$ holes, it is also moving horizontally through a row of $Z$ holes, and the design of these $B$ and $Z$ rows follows the two-dimensional design of the $Z$ and $B$ rows precisely.

Again, therefore, let $0<s_{1}<\cdots<s_{n / 2}<1$ be a series of real numbers that represent lengths of opaque portions in a $B$ row, interleaved as

$$
s_{1}, s_{n / 2}, s_{2}, s_{n / 2-1}, \ldots, s_{n / 2-1}, s_{2}, s_{n / 2}, s_{1} \text {. }
$$

This determines the $\beta$ offsets in the horizontal direction as follows (compare equation (3)):

$$
\begin{aligned}
& \beta_{i, 2 j-1}^{(0)}=\beta_{i, 2 j}^{(2)}=\left(1-s_{j}\right) / 2 \quad \text { for } j=1, \ldots, n / 2 \text {, } \\
& \beta_{i, 2 j}^{(0)}=\beta_{i, 2 j+1}^{(2)}=\left(1-s_{n / 2+1-j}\right) / 2 \quad \text { for } j=1, \ldots, n / 2-1 \text {. }
\end{aligned}
$$


By analogy with equations (4) and (5) choose

$$
\gamma_{i j}^{(0)}=\gamma_{i j}^{(2)}=s_{j} / 2-2 \alpha^{(0)}
$$

and require

$$
0<s_{\min } / 2-2 \alpha^{(0)}<s_{\max } / 2-2 \alpha^{(0)}<1
$$

This completely specifies the design of the $B$ plane in the horizontal direction, and specifies the majority of the design of the $Z$ plane in the horizontal direction.

Finally, consider the vertical motion of the ladder in the $B$ plane. It should only be possible between every other pair of holes in the first and last $B$ columns; see Fig. 7(b). This is achieved by two special-purpose columns of holes on the $Z$ plane, one for each grouping of $Z$ holes. Figure 13 shows one half of the $Z$ plane in more detail than Fig. 11; the leftmost column of holes in this figure are the special holes. Choose the vertical opaque separations between the $B$ rows to be uniform:

$$
\beta_{i j}^{(1)}=\beta_{i j}^{(3)}=\beta^{(1)}
$$

for all $i, j$. If the special purpose $Z$ holes are $Z_{1, m}, Z_{2, m}, Z_{3, m}, \ldots$, in the upper left grouping, then

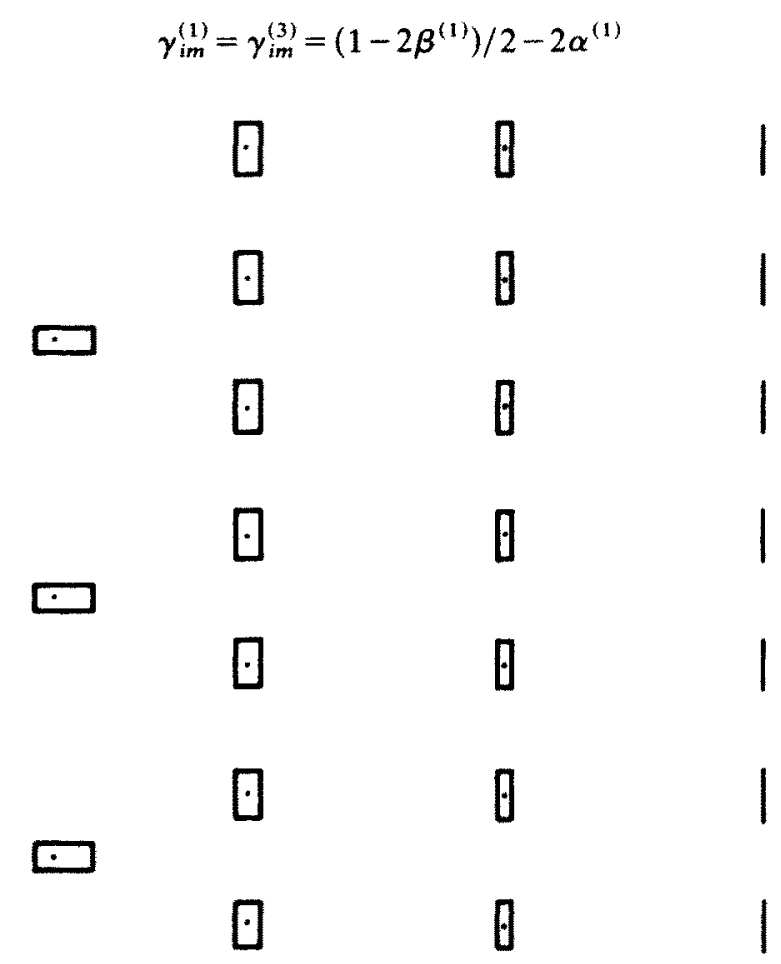

Fig. 13. Upper left half of the $Z$ plane, detail from Fig. 11. The dots are the $\zeta_{t j}$ reference points. 
and similarly for the lower right grouping. The vertical spacing for the remaining holes in the $Z$ plane is uniform.

This completes the specification of all the holes on the four planes, and all the constraint equations among their dimensions.

\subsection{Correctness of the Three-Dimensional Design}

There are five aspects to a proof of correctness of this design:

(1) The constraint equations have a solution.

(2) The ladder can move from $\left(A_{11}, B_{11}\right)$ to $\left(A_{n n}, B_{n n}\right)$ by the path $\pi$ described by Fig. 7 .

(3) No path other than $\pi$ between these two positions is possible.

(4) The number of simple moves along $\pi$ is $\Theta\left(n^{4}\right)$.

(5) The entire construction only requires $O(n)$ vertices.

Much of the correctness argument is implicit in the description of the construction, but it will be worthwhile to review it explicitly as a summary.

(1) The Equations Have a Solution. The constraint equations are equations (6)-(14). It is not at all obvious that they have a solution, as they are tightly coupled. But it is easy to check that the following assignments satisfy all equations:

$$
\begin{array}{rlrl}
\alpha^{(0)} & =\alpha^{(1)}=0.04 ; \quad \alpha^{(3)}=0.4, \\
\gamma^{(0)} & =0.4 ; & \gamma^{(1)}=0.1, \\
s_{\min }=0.2 ; & s_{\max }=0.4, \\
\beta_{\min }^{(0)}=0.3 ; & \beta_{\max }^{(0)}=0.4 ; \quad \beta^{(1)}=0.3 .
\end{array}
$$

All the figures were drawn using the sequence $s_{1}=0.2, s_{2}=0.3, s_{3}=0.4$; to obtain designs with larger values of $n$, the range between $s_{\min }$ and $s_{\max }$ can be partitioned into $n$ equal length parts.

It is important to mention that the choice of parameter values in equations (15) satisfy all the constraints inequalities "with room to spare" in the sense that the absolute value of inequalities is strictly greater than zero. We will exploit this "slack" in (5) below.

(2) The Path $\pi$ Is Possible. First consider the ladder fixed in some $B$ hole $B_{i j}$. Then equation (6) guarantees that $L$ can move horizontally through the $A$ plane as in Fig. 7(a), and equation (9) guarantees that it can move vertically when in the taller $A$ holes. Thus the path through the $A$ plane is possible.

Next consider the path through the $B$ plane. Horizontal motion is guaranteed by alignment of a $Z$ hole through $A_{11}$ or $A_{n n}$, and a choice of dimensions of the $Z$ and $B$ holes through equations (11) and (12) to yield the alternating sequence of $s_{j}$ 's as horizontal hole separations on the $B$ plane, as well as the movement permitted by the $Z$ hole. Vertical motion is guaranteed by choosing the $B$ plane 


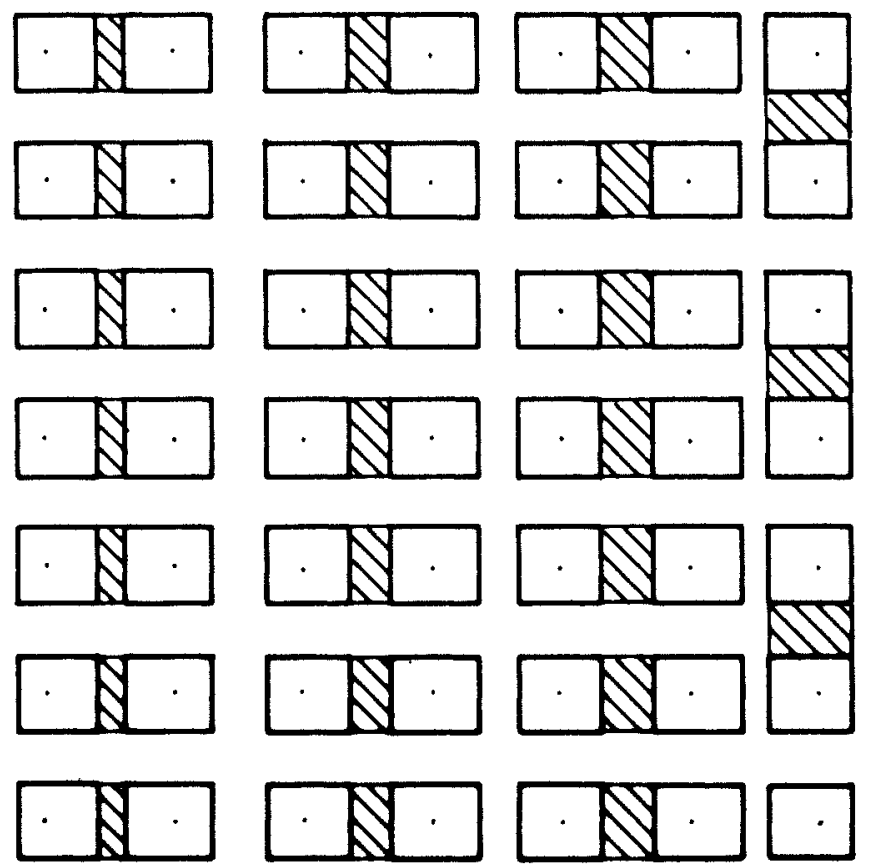

Fig. 14. Projection of $Z$ holes through $A_{11}$ onto the $B$ plane.

design uniform in the vertical direction, and by placing an extra set of holes on the $Z$ plane dimensioned according to equation (14). The placement of these special $Z$ holes restricts vertical motion to the first and last $B$ columns.

Another, perhaps more revealing, way to view these relationships is to imagine the $Z$ holes as light sources. Their projection through $A_{11}$ is shown shaded in Fig. 14: this projection, and the corresponding one through $A_{n n}$, are precisely sufficient to permit traversal by the path in Fig. 7(b). Thus the path is possible.

(3) No Other Path is Possible. We now have to show that no "shortcuts" to $\pi$ are possible. First consider motion through the $A$ plane, with the ladder fixed in some $B$ hole. Equation (7) restricts horizontal motion so that from $A_{i j}, A_{i, j \pm 2}$ cannot be reached. Thus the longest horizontal motion $L$ can make is from $A_{i, j-1}$ to $A_{i, j+1}$. Equation (8) prevents vertical motion when the ladder juts through any of the smaller $A$ holes: the latitude permitted by the $C$ holes is insufficient to reach $A_{i \pm 1, j}$ from $A_{i j}$. Equation (9) permits vertical motion for the larger $A$ holes, and equation (10) disallows vertical motion for these holes to connect more than $A_{2 i, 2}$ with $A_{2 i+1,2}$. Note that because the larger $A$ holes are larger only downward but not upward (see Fig. 8), equations (9) and (10) are only relevant for downward connections, which limits the vertical path to just that depicted in Fig. 7(a). This establishes that the path through the $A$ plane cannot be shortcut.

Now consider the path in the $B$ plane as shown in Fig. 7(b). Horizontal motion in the $B$ plane follows the same logic as the two-dimensional construction: 
equations (11) and (12) permit no motion except when $L$ is aligned through $A_{11}$ (or $A_{n n}$ ). This may be clearest when phrased in terms of the light projection analogy. Figure 14 showed the projection of lighted $Z$ holes through $A_{11}$. When projected through any other $A$ hole of the same size, the projected pattern is identical but shifted right and down. See Fig. 15. Notice that because $A_{11}$ is the only hole in the first column, the pattern must be shifted rightward; shifting only down would permit unwanted shortcuts, which is the (promised) motivation behind isolating $A_{11}$ and $A_{n n}$ in their columns. But when the pattern is shifted right and down, the vertical connections permitted by the special $Z$ holes $Z_{i m}$ move off the relevant section of the $B$ plane to the right, and the horizontal connector projections nest just as they did in the two-dimensional construction, as shown in Fig. 15. Thus the shifted pattern is useless for connections, and so permits no shortcuts. This is the key idea of the entire design.

There is a special case to consider, however. When the projection of the $Z$ holes is through one of the larger $A$ holes, the images are enlarged. But note that these $A$ holes are larger only in the vertical direction, so the enlargement is solely
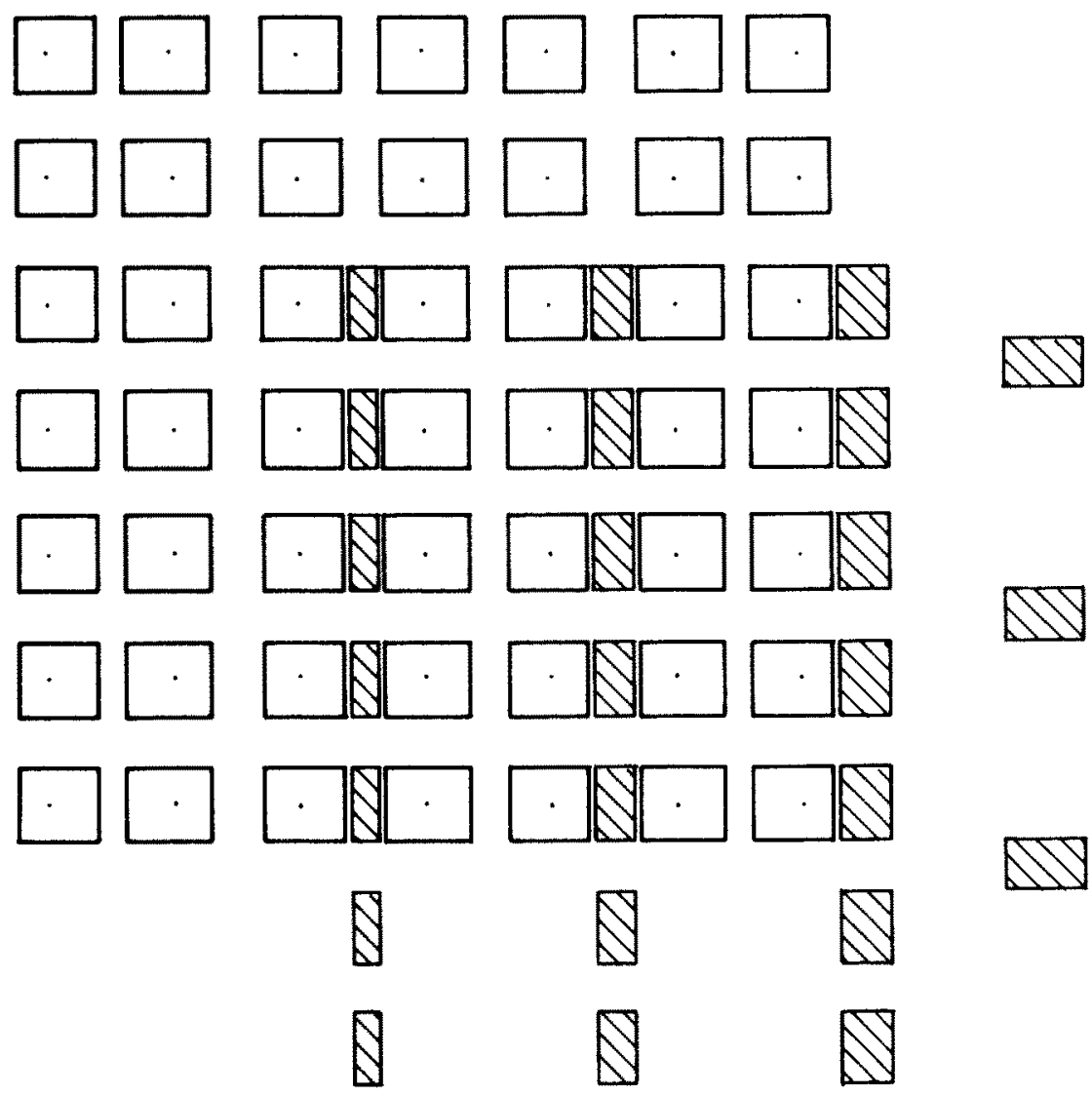

Fig. 15. Shifted projection of $Z$ holes onto the $B$ plane. 
vertical. But this just lengthens the pattern shown in Fig. 15 in the vertical direction without otherwise altering it, and it is clear that this enlargement is harmless in that it permits no shortcuts.

Vertical motion in the $B$ plane is controlled by the special $Z$ holes, which, as we have seen, only project onto the $B$ holes through $A_{11}$ or $A_{n n}$. So no other vertical motion than that shown in Fig. $7(b)$ is possible, thus showing that this path through the $B$ plane is the only one possible. And this establishes that $\pi$ is indeed the only path from $\left(A_{11}, B_{11}\right)$ to $\left(A_{n n}, B_{n n}\right)$.

(4) The Number of Simple Moves is $\Theta\left(n^{4}\right)$. Equation (6) restricts the ladder to hop at most one $A$ hole in its horizontal movement. Thus at least $2\left(n^{2} / 2\right)$ reversals of motion orthogonal to the $A$ plane are required to make one traversal of the $A$ plane. Equations (11) and (12) restrict motion through the $B$ plane to one hole per $A$ plane traversal. This leads to $\Theta\left(n^{4}\right)$ translational motion reversals from initial to final position, which requires $\Theta\left(n^{4}\right)$ simple moves for any fixed algebraic equation degree $r$.

(5) Only $O(n)$ Vertices are Required. We have to show that the $\Theta\left(n^{2}\right)$ holes on each plane can be constructed with only $O(n)$ vertices; otherwise our bound of $n^{4}$ moves might only be $N^{2}$ as a function of the input size $N$. But this is easily accomplished: the $B$ and $C$ planes are so uniform that it is obvious that $O(n)$ rectangular faces (each of four vertices) placed orthogonally in a latticework arrangement bound the desired holes. The $A$ and the $Z$ planes are a bit more complex, but they only have a linear number of nonuniformities at their boundaries, so they may still be constructed with $O(n)$ vertices.

This establishes the result when the obstacles may be faces that overlap in a plane. But if we insist that the obstacles be disjoint nondegenerate polyhedra, then an additional argument is required, as it is not possible to form truly two-dimensional holes with such obstacles.

Suppose we bound the holes by rectangular beams (each of eight vertices) of thickness $\delta>0$. Consider one hole, say $A_{i j}$, so bounded. Then the effective horizontal size of $A_{i j}$ is smaller than determined by $\alpha_{i j}^{(0)}$ and $\alpha_{i j}^{(2)}$, smaller by an amount dependent on the angle $\varphi$ by which the ladder deviates from the vertical. The shrinkage is $\varepsilon=\delta \tan \varphi$ from both sides, so that the horizontal extent of the hole is effectively

$$
a_{i j}-\alpha_{i j}^{(2)}+\varepsilon \leq x \leq a_{i j}+\alpha_{i j}^{(0)}-\varepsilon
$$

We can arrange for $\varepsilon$ to be as small as we wish by squashing the design so that the maximum value of $\varphi$ is near zero, and choosing $\delta$ small.

Now we invoke the observation that the parameters in equation (15) satisfy all constraints with "slack." Although an explicit calculation would be difficult, it should be clear that for those parameters, $\varepsilon$ may be chosen small enough so that all constraints are satisfied, albeit with less (but still nonzero) slack. For in the limit as $\varepsilon \rightarrow 0$, the equations are strictly satisfied, and the amount by which they are satisfied is continuous in $\varepsilon$ for small $\varepsilon$. 


\section{Conclusion}

We have constructed sets of obstacles composed of $O(n)$ vertices that force $\Omega\left(n^{2}\right)$ simple moves of a ladder in two dimensions, and $\Omega\left(n^{4}\right)$ simple moves in three dimensions. We have not attempted to generalize the constructions to $d$ dimensional space. A naive extrapolation of our bounds suggests that $\Omega\left(n^{2 d-2}\right)$ is the lower bound to prove.

The most interesting open problem posed by this work is to close the gap between the lower bound of $\Omega\left(n^{4}\right)$ in three dimensions and the best upper bound of $O\left(n^{5} \log n\right)$.

\section{Acknowledgments}

We are grateful to two referees for perceptive comments.

\section{References}

1. J. Canny, The complexity of robot motion planning, Ph.D. Thesis, MIT, May 1987.

2. H. Edelsbrunner, J. O'Rourke, and R. Seidel, Constructing arrangements of lines and hyperplanes with applications, SIAM J. Comput. Vol. 15, No. 2, 1986, pp. 341-363.

3. Y. Ke and J. O'Rourke, Moving a ladder in three dimensions: upper and lower bounds, Proceedings of the Third Symposium on Computational Geometry, pp. 136-145, 1987.

4. Y. Ke and J. O'Rourke, An algorithm for moving a ladder in three dimensions, Johns Hopkins Technical Report JHU/87.17, Sept. 1987.

5. D. Leven and $M$. Sharir, An efficient and simple motion-planning algorithm for a ladder moving in two-dimensional space amidst polygonal barriers, Proceedings of the First Symposium on Computational Geometry, pp. 221-227, 1985.

6. C. Ó’Dúnlaing, M. Sharir, and C. Yap, Retraction-a new approach to motion planning, Proceedings of the 15th ACM Symposium on Theory of Computing, pp. 207-220, 1983.

7. C. ÓDúnlaing, M. Sharir, and C. Yap, Generalized Voronoi diagrams for a ladder: I. Topological considerations. II. Efficient construction of the diagram, Technical Reports 139 and 140, Computer Science Department, Courant Institute, New York University, Nov. 1984.

8. J. O'Rourke, A lower bound on moving a ladder, Johns Hopkins Technical Report JHU/85-20, Nov. 1985.

9. J. T. Schwartz and M. Sharir, On the piano movers' problem: I. The case of a rigid polygonal body moving amidst polygonal barriers, Comm. Pure Appl. Math. Vol. XXXVI, pp. 345-398, 1983; also in Planning, Geometry, and Complexity of Robot Motion, edited by J. T. Schwartz, M. Sharir, and J. Hopcroft, Ablex, Norwood, NJ, 1987.

10. J. T. Schwartz and M. Sharir, On the piano movers" problem: V. The case of a rod moving in three-dimensional space amidst polyhedral obstacles, Comm. Pure Appl. Math. Vol. XXXVII, pp. 815-848, 1984; also in Planning, Geometry, and Complexity of Robot Motion, edited by J. T. Schwartz, M. Sharir, and J. Hopcroft, Ablex, Norwood, NJ, 1987.

11. M. Shatir, Presentation at the First Symposium on Computational Geometry, Baltimore, June 1985.

12. S. Sifrony and M. Sharir, A new efficient motion-planning algorithm for a rod in two-dimensional polygonal space, Proceedings of the Second Symposium on Computational Geometry, pp. 178-186, 1986.

Received June 5, 1987, and in revised form October 22, 1987. 